\title{
Pharmacological treatments prescribed to people with autism spectrum disorder (ASD) in primary health care
}

\author{
Macey L. Murray • Yingfen Hsia • Karen Glaser • \\ Emily Simonoff • Declan G. M. Murphy • \\ Philip J. Asherson • Hanna Eklund • Ian C. K. Wong
}

Received: 4 February 2013 /Accepted: 29 April 2013 /Published online: 17 May 2013

(C) Springer-Verlag Berlin Heidelberg 2013

\begin{abstract}
Rationale Autism spectrum disorders (ASDs) affect $1 \%$ of children, having significant impact on health and social outcomes. Psychotropic medication use by individuals with ASD in the USA increased over time, and polypharmacy occurred in $>50 \%$ of those prescribed. In the UK, no psychotropic drugs are approved in ASDs, and little is known about patterns of pharmacological treatment in the ASD population and associated co-morbidities.

Methods We used The Health Improvement Network, a nationally representative primary care database, to assess the prevalence of ASD diagnoses, psychotropic drug
\end{abstract}

M. L. Murray $(\bowtie) \cdot$ Y. Hsia $\cdot$ I. C. K. Wong

Centre for Paediatric Pharmacy Research, Department of Practice and Policy, UCL School

of Pharmacy, University College London, London, UK

e-mail: macey.murray@ucl.ac.uk

K. Glaser $\cdot$ H. Eklund

Department of Social Science, Health \& Medicine, King's College

London, London, UK

E. Simonoff

Department of Child and Adolescent Psychiatry, Institute of Psychiatry and Biomedical Research Centre for Mental Health,

King's College London, London, UK

D. G. M. Murphy $\cdot$ H. Eklund

Department of Forensic and Developmental Sciences, Institute of Psychiatry, King's College London, London, UK

P. J. Asherson

MRC Social, Genetic and Developmental Psychiatry Centre, Institute of Psychiatry, King's College London, London, UK

Y. Hsia • I. C. K. Wong

Centre for Safe Medication Practice and Research, Department of Pharmacology and Pharmacy, Li Ka Shing Faculty of Medicine, The University of Hong Kong, Hong Kong SAR, China prescribing and neuropsychiatric co-morbidities of 0 24 year olds between 1992 and 2008.

Results ASD prevalence increased 65-fold from $0.01 \%$ (1992) to $0.50 \%$ (2008). Psychotropic drugs were prescribed to $29 \%(1,619 / 5,651)$ of the ASD cohort; the most prescribed drugs were sleep medication $(9.7 \%$ of prescribed patients), psychostimulants (7.9\%) and antipsychotics $(7.3 \%)$. More patients were given psychostimulants and sleep medications over time from $1.5-6.3 \%$ and 2.2 $5.9 \%$ respectively. Thirty-seven per cent of the cohort had $\geq 1$ record of a neuropsychiatric co-morbidity, the most common being developmental difficulties and learning disabilities (12.6\%), behavioural, conduct and personality disorders $(11.1 \%)$ and attention deficit hyperactivity disorder $(7.5 \%)$.

Conclusions British physicians are more conservative in prescribing practice than American colleagues. However, use of psychostimulants and antipsychotics is much higher in those with ASD than in the general population. Polypharmacy was seen in $34 \%$ of prescribed patients in 2008. Additional studies examining use, efficacy, and longterm safety of antipsychotics and psychostimulants in autistic individuals are warranted.

Keywords Autistic spectrum disorder · Prevalence · Psychotropic drugs · Primary care · Co-morbidity · Children $\cdot$ Adolescents $\cdot$ Young adults

\section{Introduction}

Autistic spectrum disorders (ASDs) affect 1 in 88 children in the USA. In the UK, the prevalence of ASD is about $1 \%$ in children (Baird et al. 2006; Baron-Cohen et al. 2009). ASDs and associated co-morbid disorders significantly impact on social outcomes, education and health (Bolton et al. 1998) 
due to the early onset of ASDs, their lifelong persistence and associated pervasive impairments (Simonoff et al. 2008). The annual societal cost in the UK of supporting children with ASDs has been calculated as $£ 2.7$ billion, and these costs amount to $£ 25$ billion per annum for adults, with estimated lifetime costs of $£ 1.7$ million for those with intellectual disability and $£ 0.8$ million for those without (Knapp et al. 2009). Furthermore, individuals with ASD have a very high prevalence of co-morbid mental health conditions including attention-deficit hyperactivity disorder (ADHD), learning disabilities, oppositional or conduct disorder, emotional disorders, anxiety and other phobic disorders and chronic tic disorder (Bradley and Bolton 2006; Green et al. 2005; Simonoff et al. 2008). Hence, the development and optimisation of interventions (including behavioural treatment, educational approaches and pharmacotherapy) to alleviate symptoms/impairments of those with ASDs so as to improve the quality of life of individuals and their families is imperative.

Medication may be a helpful therapeutic intervention to manage disabling behavioural and/or mental health symptoms in the autistic population; however, there are currently no practice guidelines (Frazier et al. 2011). A review concluded that although there is no standard medication for treating ASD, people are prescribed a variety of psychotropic medications (and there is scant reliable research evidence supporting this practice in adolescents and adults) (Broadstock et al. 2007). In fact, some have suggested that individuals with ASDs experience decreased efficacy and more adverse effects from psychiatric medications such as behavioural toxicity with tricyclic antidepressants, and social withdrawal and irritability with methylphenidate (Sanchez et al. 1996; Aman et al. 1997; Handen et al. 2000). This creates uncertainty about the appropriateness of using existing pharmacological treatment(s) for comorbid conditions, such as ADHD (Frazier et al. 2011; Aman et al. 2003), and has led to recent work on drug effectiveness (Simonoff et al. 2012). Despite this uncertainty, drug surveys and studies of health insurance claims databases conducted in the USA have shown that over time more psychotropic drugs (and in particular antipsychotics, antidepressants and stimulants) are being prescribed to children, young people and adults with ASD (Aman et al. 2005; Oswald and Sonenklar 2007; Mandell et al. 2008; Esbensen et al. 2009; Williams et al. 2012). For example, in North Carolina, between 1992/1993 and 2001, psychotropic drug use rose significantly from $30.5 \%$ of children and adults with autism to $45.2 \%$. Furthermore, antidepressant use increased 3.5-fold - from $6.1 \%$ in $1992 / 1993$ to $21.4 \%$ in 2001 (Aman et al. 2005). There is also increasing evidence that the likelihood of an ASD person being prescribed medication increases with age. For instance one study reported that rates of psychotropic drug use rose from $56 \%$ of $6-11$ year olds to $73 \%$ of $18-21$ year olds
(Mandell et al. 2008). This is of importance because once an individual has been prescribed a psychotropic medication, they are (a) 11 times more likely to remain on it than for non-psychotropic drugs (Esbensen et al. 2009) and (b) much more likely to be exposed to (potentially hazardous) polypharmacy (e.g. some have reported that more than half of children and youths with ASD who were prescribed psychotropic medication experienced this (Logan et al. 2012; Frazier et al. 2011).

Although there is limited evidence to guide psychotropic medication use in the ASD population, two drugs have shown efficacy for the alleviation of behavioural symptoms in children and adolescents with autistic disorder: risperidone (Research Units on Pediatric Psychopharmacology Autism Network (RUPPAN) 2002; Shea et al. 2004) and aripiprazole (Owen et al. 2009; Marcus et al. 2009). In 2006, risperidone was approved by the Food and Drug Administration (FDA) in the USA for the treatment of irritability associated with autistic disorder in 5-16 year olds; this includes aggression and deliberate selfinjuriousness. Aripiprazole was also approved by the FDA in 2009 after demonstrating efficacy in the same indication in 6-17 year olds (Owen et al. 2009; Marcus et al. 2009). An application for UK approval was submitted but subsequently withdrawn by the licence holder of risperidone, JanssenCilag, in 2006 after receiving an offer of conditional approval - but limiting its indication to the symptomatic treatment of severe aggression and violence in children with autism (Morgan \& Taylor 2007). Safety monitoring through a treatment registry formed part of the conditions due to concerns that risperidone could be misused as long-term chemical control in children. In the UK, risperidone remains unlicensed for use in children with autism, but it is approved for the treatment of persistent aggression in conduct disorder in children of sub-average intellectual functioning. The reported side effects of antipsychotic drugs include significant weight gain and other metabolic effects such as hyperprolactinaemia and diabetes mellitus (Almandil and Wong 2011; Almandil et al. 2013), and movement disorders such as tardive dyskinesia, tremor and dystonia (Almandil and Wong 2011; Fleischhaker et al. 2006; Robb et al. 2011); life-threatening side effects such as rhabdomyolysis, neuroleptic malignant syndrome and seizures have also been reported (Star et al. 2012; Rani et al. 2009, 2011). However little is known about the long-term safety of antipsychotics, and polypharmacy with other drug classes, especially in the autistic population. This is a valid concern because of the early diagnosis of ASDs and the lifetime persistence of symptoms, which are increasingly managed with psychotropic drugs (Esbensen et al. 2009).

Studies have identified substantial support needs for ASD in terms of health, social care and educational services (Knapp et al. 2009; Barrett et al. 2012), but to date, we have 
very little data in the UK about the prescribing of pharmacological treatment by young people with ASDs and associated neuropsychiatric co-morbid disorders. Information about prescribing practices and co-morbidities is necessary for prioritising further research into the efficacy and shortand long-term safety of psychotropic medications in this particular patient population. Hence, we conducted an observational cohort study based on a primary care database to investigate the pharmacological treatments used among a cohort of children, adolescents and young adults diagnosed with ASD.

\section{Method}

\section{Study design}

A descriptive cohort study was conducted using The Health Improvement Network (THIN) database to investigate the incidence and prevalence of ASD diagnoses, psychotropic medication prescribing and neuropsychiatric related comorbidities of children, adolescents and young adults in UK primary care.

\section{Data source}

THIN contains anonymised computerised information systematically recorded by general practitioners (GPs) in the UK for patient management. The database provider collates and organises this information in order for it to be used for research. In the UK, almost all patient care is managed by GPs in primary care, who act as gatekeepers of the UK National Health Service. When required, GPs will refer patients to hospital consultants or specialists in secondary care for diagnosis and initiation of treatment, and GPs usually continue to monitor their patients and issue prescriptions. The diagnosis and management of ASDs and the initiation of drug treatment for symptoms of ASDs (or co-morbidities) is usually the responsibility of specialist teams, and general practitioners manage patients in primary care through shared care arrangements with these teams.

THIN covers approximately $5.7 \%$ of the UK population with 3.6 million active patients from 464 general practices (Cegedim Strategic Data Medical Research UK 2009). The demographic distribution of THIN is broadly representative of that of the UK; therefore, analysis of the clinical and prescribing data will provide information on trends that is representative of national trends. This is particularly useful in evaluating treatment rates, rates of specific diagnoses and changes in these rates.

THIN is a rich source of clinical primary care data and contains information on patient demographics, prescriptions, diagnoses and referrals. Drugs are coded in the database using MULTILEX ${ }^{\circledR}$ codes (First DataBank; THIN). Diagnoses, symptoms and referrals are coded using Read Clinical Terms, a comprehensive hierarchical system (Chisholm 1990). The database has been previously used to study trends in disease incidence and prescribing patterns in paediatric and adult populations (McCarthy et al. 2012a,b; Wijlaars et al. 2012). A previous study using a similar UK primary care database (the former General Practice Research Database; GPRD) has validated diagnoses of ASDs in GP records by expert review and by a computerised diagnostic algorithm of Diagnostic and Statistical Manual of Mental Disorders (DSM)-IV symptoms ratings, showing they are appropriate for the identification of ASD cases (Fombonne et al. 2004). Practices who contribute to THIN and/or GPRD use the same practice management software (Vision, In Practice Systems Ltd), so data from both databases are collected in a similar manner and structure; in fact a large proportion $(66 \%, 327$ of 495 practices in 2001-2008) of practices contributing to THIN also contribute to GPRD (Cai et al. 2012).

\section{Study population}

The study population comprised all individuals in THIN who were children, adolescents and young adults aged $<25$ years who had a record of diagnosis of ASD in the study period between 1 January 1992 and 31 December 2009. The start date of each patient was defined as the latest of the following: the date of the patient's registration at the general practice; the date that the general practice began using the Vision software (a clinical management system); and the date that the practice was deemed to meet a key quality indicator known as the Acceptable Mortality Reporting (Maguire et al. 2009). Patients were included if they had an observation period of least 6 months available from their start date and were registered with the general practice during the study period.

\section{Prevalence of ASD}

Patient records dated within one year after a patient's start date (which may or may not be within the study period) were screened to identify diagnoses of ASD. Any individual with an ASD diagnosis within 1 year of the start date were considered affected with ASD (i.e. prevalent) unless they were aged $<2$ years at the time of the diagnosis. Autistic disorder can be diagnosed in children as young as 2 years of age, whereas those with Asperger disorder and pervasive development disorder-not otherwise specified are frequently not reliably diagnosed until 4-5 years of age (Shattuck et al. 2009). Hence, patients with a record of ASD at ages $<2$ years are likely to be incident with ASD. Incident patients were defined as (1) those who have a first 
diagnosis of ASD following the first year screening period and (2) those with an ASD diagnosis when aged $<2$ years during the screening period. The index date for each patient was defined as the date of the first recorded diagnosis of ASD following the patient's start date. The annual prevalence of ASD was calculated by counting all patients incident with ASD in a particular year and all prevalent patients in active follow-up of that year divided by the total number of individuals in the THIN mid-year population (i.e., those who remained registered on the database on 1st July of that calendar year). The annual incidence of ASD was calculated by counting all patients incident with ASD in a particular year divided by the total number of individuals in the THIN mid-year population. Annual prevalence of ASD was calculated by age group defined as $0-5$ year olds (young children), 6-12 year olds (children), 13-17 year olds (adolescents) and 18-24 year olds (young adults).

\section{Drug prescribing}

Nine categories of drugs were defined (by MLM, ES and $\mathrm{KG}$ ) as: stimulants (methylphenidate, dexamfetamine and atomoxetine), antidepressants, antipsychotics, antiepileptics/mood stabilisers, benzodiazepines, sleep medication (including melatonin), clonidine, beta-blockers and antiparkinsonism drugs that may be co-prescribed with antipsychotics to counteract unwanted motor effects. The prescriptions for the study drugs of each patient recorded on or after their index date were identified, and the annual proportions of the ASD cohort prescribed drug treatment were calculated by drug category and by individual drug (with $95 \%$ confidence intervals).

\section{Co-morbidities}

The clinical records of each patient recorded on or after their index date were screened for neuropsychiatric co-morbidities. Diagnostic codes (Read codes) were categorised into eleven groups (by DGM, ES and MLM,): psychotic disorders (including schizophrenia and delusions); behavioural, conduct and personality disorders; mood disorders; anxiety and phobias (including obsessive-compulsive disorder); ADHD; suicidal behaviour and self-harm; anorexia and bulimia; tic disorders; developmental difficulties and learning disabilities; substance and alcohol misuse. The proportions of the cohort with neuropsychiatric comorbidities were calculated.

Data management and analyses were performed using Stata SE version 11.2 (StataCorp, College Station, TX, USA).

The study protocol was granted approval by the Scientific Review Committee of Cegedim Strategic Data (CSD) Medical Research UK, the data providers of THIN.

\section{Results}

Characteristics of the ASD cohort

There were 5,651 patients aged under 25 years in the THIN database with at least one diagnosis record of ASD during the study period; $83.0 \%$ were male $(n=4,688)$. Table 1 provides details on the characteristics of the study cohort. In 4,541 patients who became incident with ASD during the study period, the mean age at first recorded diagnosis was 9.0 years (SD: 4.74 years); however, a bimodal distribution of age was observed with peak frequencies of first diagnoses recorded at 4 and 8 years of age. First diagnoses of ASD were recorded at a later age in female than in male.

Prevalence/incidence of ASD diagnoses

Overall prevalence of ASD diagnoses during the study period was 2.23 persons per 1,000 mid-year population (MYP); the highest prevalence was seen in children with 3.87 children per 1000 MYP. In 2008, ASD prevalence for young children was 2.06 per 1,000 MYP, 8.89 per 1,000 MYP in children, 6.64 per 1,000 MYP in adolescents (13-17 years) and 2.44 per 1,000 MYP in young adults (18-24 years).

Figure 1 shows the prevalence of ASD in the cohort over the study period. The prevalence of ASD increased 64.6fold from 0.08 persons per 1,000 MYP in $1992(95 \% \mathrm{CI}$, $0.05-0.12)$ to 5.04 persons per 1,000 MYP in 2008 (95\% CI, 4.89-5.19). The increase was greater in male (77.1-fold) than in female (36.8-fold). The incidence of ASD also rose in the same period by 23.7 -fold from 0.03 persons per 1,000 MYP in 1992 (95\% CI, 0.01-0.05) to 0.67 persons per 1,000 MYP in 2008 (95\% CI, 0.61-0.72). The rise in incidence was greater in female (32.9-fold) than male (23.1-fold).

\section{Drug prescribing}

There was a total of 1,619 patients $(28.7 \%$; $95 \%$ CI, $27.3-$ $30.1 \%$ ) of the cohort who received 47,738 prescriptions for the study drugs; proportionally, more female patients were prescribed these drugs than male (Table 1). In 2008, $33.8 \%$ of those prescribed psychotropic medications (305 of 902 patients) were given prescriptions for two or more drugs. The three most commonly prescribed drug groups overall (and in male patients) were sleep medication [9.7\% overall (95\% CI, 8.9-10.6\%), $10.0 \%$ of male patients (95\% CI, 9.1-10.9\%)], stimulants [7.9\% overall (95\% CI, $7.2-$ $8.7 \%), 8.7 \%$ of male patients (95\% CI, 7.8-9.5\%)] and antipsychotics [7.3\% overall (95\% CI, 6.7-8.1\%), $7.0 \%$ of male patients (95\% CI, 6.3-7.8\%)]; for female patients, they were antiepileptics/mood stabilisers $(11.0 \%$; $95 \% \mathrm{CI}$, 
Table 1 Characteristics of the patient cohort with autistic spectrum disorder (ASD) diagnoses

\begin{tabular}{|c|c|c|c|}
\hline Cohort characteristics & All & Male & Female \\
\hline Number of subjects aged $0-24$ years (\%) with at least one diagnosis of ASD & $5,651(100.0)$ & $4,688(83.0)$ & $963(17.0)$ \\
\hline Mean age (SD) at first recorded diagnosis of ASD (years) & $9.3(5.05)$ & $9.2(4.92)$ & $10.1(5.60)$ \\
\hline Median age (IQR) at first recorded diagnosis of ASD (years) & $8.4(7.24)$ & $8.2(6.92)$ & $9.1(8.64)$ \\
\hline \multicolumn{4}{|l|}{ ASD diagnoses } \\
\hline Total number of ASD diagnoses & 8,602 & 7,205 & 1,397 \\
\hline Mean number of ASD diagnoses per person (SD) & $1.5(1.25)$ & $1.5(1.27)$ & $1.5(1.10)$ \\
\hline Median number of ASD diagnoses per person (IQR) & $1.0(1.00)$ & $1.0(1.00)$ & $1.0(1.00)$ \\
\hline \multicolumn{4}{|l|}{ Prevalent/incident patients } \\
\hline $\begin{array}{l}\text { Number of subjects prevalent with ASD (ASD diagnosis recorded } \\
\text { within one year of entry into study) }\end{array}$ & $1,110(19.6)$ & $919(19.6)$ & $191(19.8)$ \\
\hline Number of subjects incident with ASD & $4,541(80.4)$ & $3,769(80.4)$ & $772(80.2)$ \\
\hline Incident patients: mean age (SD) at first recorded diagnosis of ASD (years) & $9.0(4.74)$ & $8.9(4.61)$ & $9.6(5.29)$ \\
\hline Incident patients: median age (IQR) at first recorded diagnosis of ASD (years) & $8.1(6.72)$ & $8.0(6.44)$ & $8.5(8.08)$ \\
\hline Incident patients: mean number of ASD diagnoses per person (SD) & $1.5(1.33)$ & $1.5(1.34)$ & $1.4(1.28)$ \\
\hline Incident patients: median number of ASD diagnoses per person (IQR) & $1.0(1.00)$ & $1.0(1.00)$ & $1.0(1.00)$ \\
\hline \multicolumn{4}{|l|}{ Prescribing } \\
\hline Total number of prescriptions prescribed post-index date & 158,285 & 122,587 & 35,698 \\
\hline Number of study drug prescriptions issued at any time post-index date & 47,738 & 36,983 & 10,755 \\
\hline Total number of patients (\%) prescribed at least one prescription for a study drug & $1,619(28.6)$ & $1,312(28.0)$ & $307(31.9)$ \\
\hline
\end{tabular}

9.0-13.3\%), antidepressants (9.0\%; $95 \%$ CI, 7.2-11.1\%), and sleep medication $(8.5 \%$; $95 \%$ CI, 6.8-10.6\%). Clonidine and beta-blockers were rarely prescribed; only 30 and 36 patients in the total cohort respectively were prescribed these drugs. The five most commonly prescribed drugs were methylphenidate $(7.1 \%$ of the ASD cohort; $95 \%$ CI, 6.4-7.8 \%), melatonin (5.7\%; $95 \%$ CI, 5.1$6.4 \%)$, risperidone $(5.6 \%$; $95 \%$ CI, 5.0-6.3\%), diazepam (3.2\%; $95 \%$ CI, 2.8-3.7\%) and valproic acid [3.2\%; $95 \%$ CI, 2.8-3.7\%].

Stimulants accounted for $23.0 \%(n=10,981)$ of all psychotropic drug prescriptions issued to the cohort, and the average number of prescriptions per patient was 24.6. However, the drug group with the most prescriptions was antiepileptics/mood stabilisers with $33.2 \%$ of all prescriptions $(n=15,868)$; the mean number of prescriptions per patient was 44.1 (Table 2).

The numbers of individuals with ASD diagnoses between 1992 and 1998 were relatively low (Fig. 1), which made prescribing rates unstable. Consequently, Fig. 2 shows the prescribing rates of the drug groups from 1999 to 2008 only.

The proportion of the cohort prescribed stimulants rose by $4.7 \%$ between 1999 (1.5\%; $95 \%$ CI, 0.7-2.9\%) to $6.3 \%$ (95 \% CI, 5.6-7.0\%) in 2008 (Fig. 2a). Sleep
Fig. 1 Prevalence of autistic spectrum disorder (ASD) in individuals aged $0-24$ years (with $95 \%$ confidence intervals)

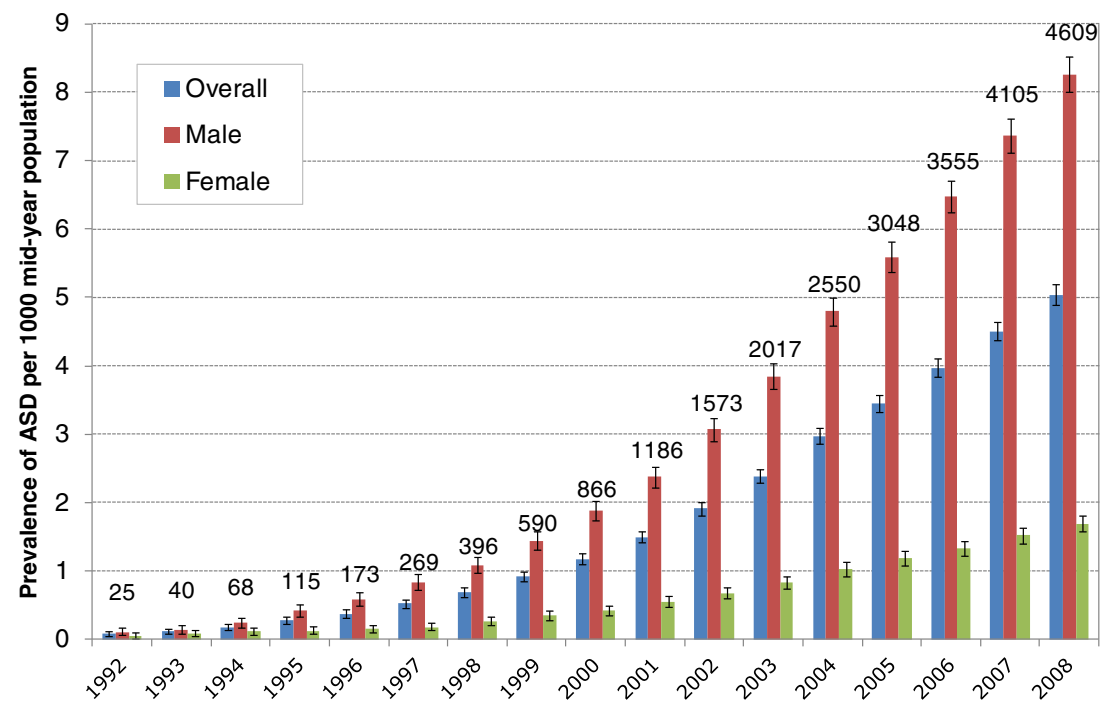




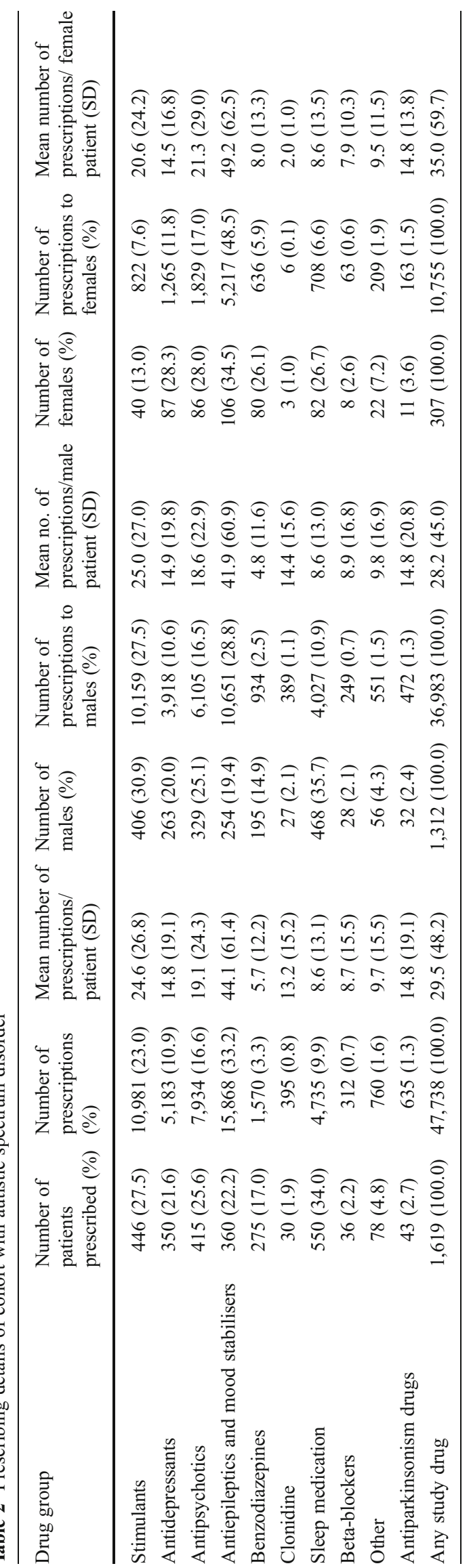

medications also were prescribed to $3.7 \%$ more young people during the same period [2.2\% $(95 \% \mathrm{CI}, 1.2-$ $3.8 \%$ ) in 1999 to $5.9 \%$ (95\% CI, 5.2-6.6\%) in 2008]. The prescribing of benzodiazepines, antipsychotics, and antiparkinsonism drugs remained stable (Figs. 2a, b), and a slight downward trend in antidepressant use was seen (Fig. 2c). There was a $1.8 \%$ decline in the proportion of patients prescribed antiepileptic drugs between 1999 and 2008 , from $6.1 \%$ (95\% CI, $4.3-8.4 \%)$ to $4.3 \%$ (95\% CI, 3.7-4.9\%; Fig. 2c).

Psychiatric co-morbidities

There were 2,062 patients (36.5\% of cohort; $95 \%$ CI, 34.9$38.1 \%$ ) who had at least one record of a neuropsychiatric comorbidity on or after their first recorded diagnosis of ASD on the database. The most common neuropsychiatric comorbidities were developmental difficulties and learning disabilities (12.6\% of the cohort; $95 \%$ CI, $11.7-13.6 \%$ ), behavioural, conduct and personality disorders $(11.1 \%$; $95 \% \mathrm{CI}, 10.3$ $12.0 \%)$, ADHD (7.5\%; $95 \%$ CI, 6.8-8.2 \%), and epilepsy (5.0\%; $95 \%$ CI, 4.4-5.6\%; Table 3). Of those with an ADHD diagnosis, $62.0 \%(n=261)$ were prescribed a psychostimulant during the study period, and $21.4 \%(n=90)$ received at least one prescription for a sleep medication. Furthermore, $41.5 \%$ $(185 / 446)$ of those given at least one psychostimulant prescription had no co-morbidity of ADHD recorded during the period. Almost three quarters of patients $(n=210)$ with epilepsy recorded were prescribed an antiepileptic drug.

\section{Discussion}

To our knowledge, this is the first naturalistic study of psychotropic drug prescribing and neuropsychiatric comorbidities in a large nationally representative cohort of ASD patients in UK primary care. The database has provided rich longitudinal data to enable us to characterise the autistic population and the prescribing practices of GPs undertaking their clinical care (usually by shared care arrangements with secondary care physicians).

We found the incidence and prevalence of ASD have substantially increased 23.7- and 64.6-fold, respectively, over the period between 1992 and 2008. These increases may be due to improved recognition and diagnosis of ASD, broadened diagnostic criteria and increased awareness and acceptance of ASD by healthcare professionals and parents (Baird et al. 2006; Baron-Cohen et al. 2009; Simonoff 2012). The 2008 prevalence of ASD diagnoses in 6-12 year olds of our cohort $(0.89 \%$; $95 \%$ CI, $0.85-0.93 \%)$ is comparable to that reported by Baird et al. (2006) (1.16\%; $95 \%$ CI, 0.90-1.42\%) from their Special Needs and Autism Project sample (SNAP) and the estimate of $1.57 \%$ 
Fig. 2 Proportion of cohort with autistic spectrum disorder (ASD) prescribed: a stimulants, sleep medication or benzodiazepines;

b antipsychotic

or antiparkinsonism drugs;

c antidepressants

or antiepileptics and mood stabilisers (1999-2008)
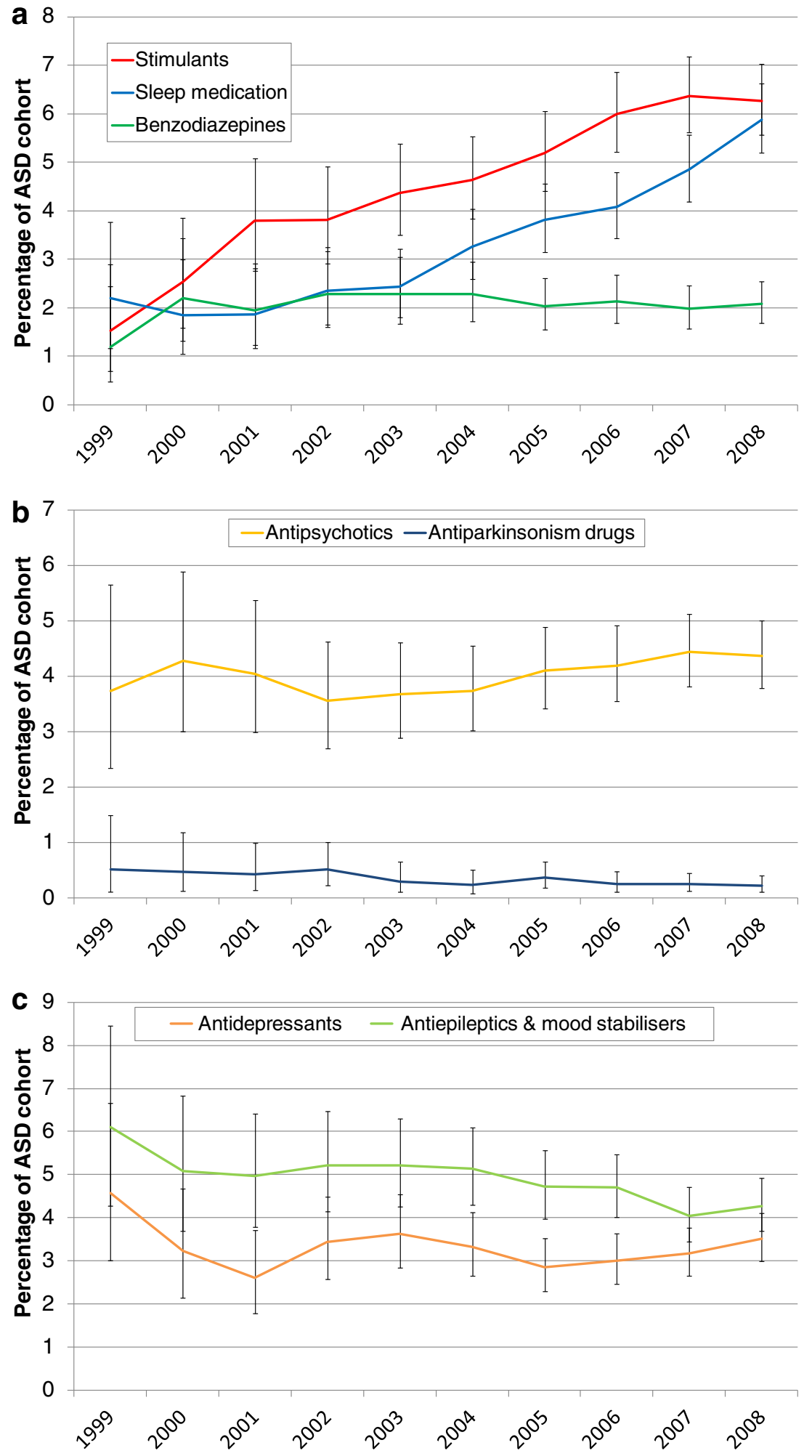

(95\% CI, 0.9-2.46\%) in a Cambridgeshire school-based population (Baron-Cohen et al. 2009).

There was more conservative use of psychotropic drugs by British GPs (usually initiated by specialist physicians) compared with the practices of their American colleagues.
Only $29 \%$ of our cohort (26\% aged 0-21 years) received psychotropic drug prescriptions in UK primary care. In contrast, Mandell et al. (2008) found $56 \%$ of Medicaid-enrolled youths with ASDs aged 0-21 years received at least one psychotropic drug in 2001; likewise, in 2002, $57 \%$ of under 
Table 3 Neuropsychiatric comorbidities of the cohort with autistic spectrum disorder

\begin{tabular}{lccc}
\hline Neuropsychiatric co-morbidities & All & Male & Female \\
\hline Attention deficit hyperactivity disorder & $421(7.5)$ & $378(8.1)$ & $43(4.5)$ \\
Anorexia and bulimia & $47(0.8)$ & $30(0.6)$ & $17(1.8)$ \\
Anxiety and phobias (including obsessive & $251(4.4)$ & $192(4.1)$ & $59(6.1)$ \\
$\quad$ compulsive disorder) & $628(11.1)$ & $505(10.8)$ & $123(12.8)$ \\
Behavioural, conduct and personality disorders & $189(3.3)$ & $134(2.9)$ & $55(5.7)$ \\
Mood disorders & $283(5.0)$ & $209(4.5)$ & $74(7.7)$ \\
Epilepsy & $713(12.6)$ & $562(12.0)$ & $151(15.7)$ \\
Developmental difficulties and learning disabilities & $37(0.7)$ & $29(0.6)$ & $8(0.8)$ \\
Psychotic disorders (including schizophrenia and delusions) & $18(0.3)$ & $13(0.3)$ & $5(0.5)$ \\
Substance and alcohol misuse & $63(1.1)$ & $45(1.0)$ & $18(1.9)$ \\
Suicidal behaviour and self-harm & $64(1.1)$ & $60(1.3)$ & $4(0.4)$ \\
Tic disorders & $287(5.1)$ & $227(4.8)$ & $60(6.2)$ \\
Unclassified neuropsychiatric disorders & $2,062(36.5)$ & $1,669(35.6)$ & $393(40.8)$ \\
Any neuropsychiatric co-morbidity & & & \\
\hline
\end{tabular}

21s enrolled to another US claims database (MarketScan ${ }^{\circledR}$ ) were prescribed psychotropic drugs (Oswald and Sonenklar 2007).

Psychotropic drug prescribing practices will be strongly influenced by co-morbidities associated with ASDs, which may explain the large proportion of those prescribed psychotropic drugs who were given more than one psychotropic drug (34\% of prescribed patients in 2008). A third of our cohort had at least one neuropsychiatric co-morbidity recorded in their primary care records following ASD diagnosis, the most common being developmental difficulties and learning disabilities, behavioural, conduct and personality disorders and ADHD. Simonoff et al. (2008) found in their SNAP cohort that $28.2 \%$ were co-morbid with ADHD, $30.0 \%$ had oppositional or conduct disorder and $41.9 \%$ had anxiety or phobias; our cohort had much lower proportions of these co-morbidities. It is possible that there was underrecording as specialist physicians may find it difficult to diagnose co-morbid psychiatric disorders due to communication impairment or cognitive problems in individuals (Leyfer et al. 2006). Alternatively, there may be nosological conventions, which exclude the diagnosis of certain symptoms as co-morbid psychiatric disorders, for example, symptoms of hyperactivity and impulsiveness in autistic people may not be reported as ADHD (RUPPAN 2005; White et al. 2009). New diagnostic guidelines in DSM-5 and almost certainly International Classification of Diseases 11 th revision will remove the exclusion of making a diagnosis of ADHD in the context of ASD, which has been present. Therefore, a large proportion of stimulant prescribing in our study is likely to be for the treatment of co-morbid ADHD or symptoms of hyperactivity (Simonoff et al. 2008; Frazier et al. 2011), even though two fifths of stimulant-prescribed patients did not have an ADHD diagnosis recorded during the study period.
We found that individuals with ASD are much more likely to be prescribed psychostimulants in comparison with the UK general population. For example, McCarthy et al. (2012a) reported that 0.92 and $0.74 \%$ of children aged 6-12 years and 13-17 years, respectively, in THIN were prescribed a stimulant drug in 2008. However, $6.60 \%$ (of 6-12 years olds) and 8.79\% (13-17 years) of our cohort received at least one prescription for methylphenidate, dexamfetamine or atomoxetine in the same year. Thus, compared with a child in the general population, a child with ASD is seven times more likely to be prescribed a psychostimulant. For autistic adolescents, psychostimulant prescribing is almost 12 times higher than that of the general population.

The increase in prescribing of stimulants over time may reflect the increasing recognition of co-morbid ADHD (and its persistence) in young people with ASDs. In addition, secondary care physicians may be more willing to initiate (and general practitioners more willing to prescribe) methylphenidate following evidence that it is effective in some autistic young people with ADHD (RUPPAN 2005).

Two thirds of the autistic population are reported as having moderate sleep disturbances (Souders et al. 2009); however, $<10 \%$ of our cohort received sleep medications ( $<5 \%$ were prescribed benzodiazepines, some of which may be used to alleviate sleep problems); it is likely that UK GPs only consider to prescribe pharmacological treatments to patients with severe sleeping disorders. It is interesting to note the rising trend of sleep medication prescribing. The trend may be due to increased diagnosis of co-morbid ADHD and/or the use of sleeping medications to counteract the effects of stimulants on sleep.

Prescribing for antipsychotics and antidepressants in the cohort was relatively stable between 1999 and 2008, at the overall prescribing rates of $4.1 \%$ (95\% CI, 3.85-4.37\%) 
and $3.3 \%$ (95\% CI, 3.03-3.49\%) respectively. This differs to reported increases in antidepressant and antipsychotic use in the American autistic population. Two drug surveys conducted in North Carolina in 1992/1993 and 2001 revealed a significant 3.5 -fold increase in the proportion of children and adults with autism prescribed antidepressants (Aman et al. 2005). Williams et al. (2012) found that use of antipsychotics was high (34\% in 2008 compared to $7 \%$ of our cohort) in their study of Kentucky-based children with autism. This was attributed to the greater use of atypical antipsychotics following the licensing of risperidone in the USA for the treatment of irritability associated with autistic disorder (Williams et al. 2012). Although risperidone remains unlicensed in the UK for this indication, we still found a fifth of the cohort who were prescribed psychotropic drugs were given risperidone. While the ratio of the cohort prescribed antipsychotics has remained relatively stable between 1999 and 2008, a high proportion of children and teenagers with ASD are prescribed antipsychotics in comparison with the general British population. In 2005, the prevalence of antipsychotic prescribing in $0-18$ year olds is reported to be $0.08 \%$ (Rani et al. 2008) compared to $2.98 \%$ of the same age group in our cohort (a 38.7-fold increased risk).

Antiparkinsonism drugs were prescribed to a very small proportion of patients $(0.8 \%$ of ASD cohort, $n=43)$ probably to mitigate extrapyramidal symptoms of antipsychotics as has been reported elsewhere (Esbensen et al. 2009).

There may be some underestimation of prescribing as some patients may be treated in specialist centres and THIN does not systematically record prescriptions generated in non-primary care healthcare settings (some information on drug treatment may be recorded in discharge summaries and hospital letters). Furthermore, some GPs may not participate in shared care prescribing arrangements, especially of psychotropic drugs in off-label use and controlled drugs such as the psychostimulants - methylphenidate and dexamfetamine. The database does not record treatment compliance or dispensing of prescriptions. There may be underestimation of neuropsychiatric co-morbidities due to the censoring of patient records, or incomplete transcription of clinical details from correspondence with specialist teams. Lastly, prescriptions are not directly linked to diagnoses in THIN, so we cannot definitely identify indications of use.

Further assessment of the efficacy and short- and longterm safety of psychotropic drugs for the treatment of behavioural problems in this population is urgently required as only two drugs so far, risperidone and aripiprazole, have shown efficacy (RUPPAN 2002; Shea et al. 2004; Owen et al. 2009; Marcus et al. 2009). More recently, optimal dosing of methylphenidate was shown to be effective in reducing ADHD symptoms in about $40 \%$ of children with intellectual disability (including those with ASDs), and the adverse effects profile was similar to that seen in typically developing children (Simonoff et al. 2012). Safety is an important consideration across all ages due to the lifetime persistence of symptoms and impairment, polypharmacy of psychotropic medications and the difficulties that some individuals may have in communicating about adverse effects. More information on psychotropic drug use (and the appropriateness of prescribing for co-morbidities) in the ASD population across Europe would be beneficial to focus European drug research activities and to inform clinical practice. Furthermore, there is a need to prioritise research of the most commonly prescribed drugs in the autistic population: the antipsychotics (in particular risperidone safety), the psychostimulants (especially efficacy and safety in those co-morbid with ADHD) and sleep medications.

\section{Conclusions}

The increase in prevalence of ASD diagnoses recorded in UK primary care between 1992 and 2008 may be due to improved recognition of ASDs, widening diagnostic criteria and more awareness and acceptance of the conditions. However, there is under-reporting of neuropsychiatric co-morbidities in the British autistic population following diagnosis with ASDs, possibly due to diagnostic exclusion or communication difficulties in patients. Less than a third of individuals with ASD receive at least one psychotropic drug prescription, which are mostly for stimulants, antiepileptic drugs and mood stabilisers and antipsychotics. Of those who are prescribed psychotropic medication, a fifth is treated with risperidone, and at least a third received more than one drug. Hence, further research into the appropriate use, efficacy and long-term safety of antipsychotics and stimulants in the autistic population is warranted to support clinical practice for optimal and safe treatment of ASDs and their co-morbidities.

Acknowledgements We thank the contributing general practitioners of the THIN database, and the data providers, CSD Medical Research UK for their kind assistance.

Funding The use of the THIN data was funded by the National Institute for Health Research Programme Grant for Applied Research: crossing the divide, effective treatments for people with neurodevelopmental disorders across the lifespan and intellectual ability. The research leading to these results has received support from the Innovative Medicines Initiative (IMI) Joint Undertaking under grant agreement no. 115300: European Autism Interventions (EU-AIMS), resources of which are composed of financial contribution from the European Union's Seventh Framework Programme (FP7/2007-2013) and EFPIA companies in kind contribution. 
Conflicts of interest YH, KG and HE have no conflicts of interest. MLM has received research funding from Shire and Pfizer; the present work is unrelated. She is currently receiving funding from the European Union's Seventh Framework Programme to investigate the long-term safety of methylphenidate in individuals with ADHD [attention deficit hyperactivity disorder drugs use chronic effects (ADDUCE)], the efficacy and safety of risperidone in children with conduct disorder [Pediatric European Risperidone Studies (PERS)] and the association between suicide-related adverse events and risperidone, fluoxetine and montelukast [Suicidality: Treatment Occurring in Paediatrics (STOP)]. ES has received research funding from The Wellcome Trust and The Health Foundation. DGM has received research funding and honoraria from various pharmaceutical companies, including Shire and Janssen-Cilag. He is currently receiving funding from the IMI for EU-AIMS to identify new treatment targets for ASD. PA has acted in an advisory role for Shire, Janssen-Cilag, Eli Lilly and Flynn Pharma. He has received education or research grants from Shire, Janssen-Cilag and Eli-Lilly. He has given talks at educational events sponsored by the above companies. IW has received research funding and honoraria from various pharmaceutical companies, including, Shire, Janssen-Cilag and Bristol-Myers Squibb. He is currently receiving funding from the European Union's Seventh Framework Programme to investigate the safety of risperidone in children (PERS) and the long-term safety of methylphenidate (ADDUCE). He is also a director of Healthcare Innovation Technology Service Limited, which received funding from the IMI for taking part in the EU-AIMS project.

\section{References}

Almandil NB, Liu Y, Murray ML, Besag FMC, Aitchison KJ, Wong ICK (2013) Weight gain and other metabolic adverse effects associated with atypical antipsychotic treatment of children and adolescents: a systematic review and meta-analysis. Paediatr Drugs 15(2):139-150

Almandil NB, Wong IC (2011) Review on the current use of antipsychotic drugs in children and adolescents. Arch Dis Child Educ Pract Ed 96(5):192-196

Aman MG, van Bourgondien ME, Osborne PW, Sarphare G (1997) Side effects associated with psychoactive medication in individuals with autism. J Autism Dev Disord 27(3):342-344

Aman MG, Lam KS, Van Bourgondien ME (2005) Medication patterns in patients with autism: temporal, regional, and demographic influences. J Child Adolesc Psychopharmacol 15(1):116-126

Aman MG, Buican B, Arnold LE (2003) Methylphenidate treatment in children with borderline IQ and mental retardation: analysis of three aggregated studies. J Child Adolesc Psychopharmacol 13(1):29-40

Baird G, Simonoff E, Pickles A, Chandler S, Loucas T, Medrum D et al (2006) Prevalence of disorders of the autism specturm in a population cohort of children in South Thames: the Special Needs and Autism project (SNAP). Lancet 368(9531):210-215

Baron-Cohen S, Scott FJ, Allison C, Williams J, Bolton P, Matthews FE, Brayne C (2009) Prevalence of autism-spectrum conditions: UK school-based population study. Br J Psychiatry 194(6):500-509

Barrett B, Byford S, Sharac J, Hudry K, Leadbitter K, Temple K, Aldred C, Slonims V, Green J; PACT Consortium (2012) Service and wider societal costs of very young children with autism in the UK. J Autism Dev Disord 42(5):797-804

Bolton PF, Pickles A, Murphy M, Rutter M (1998) Autism, affective and other psychiatric disorders: patterns of familial aggregation. Psychol Med 28:385-395

Bradley E, Bolton PF (2006) Episodic psychiatric disorder in teenagers with learning disabilities with and without autism. Br J Psychiatry 189:361-366
Broadstock M, Doughty C, Eggleston M (2007) Systematic review of the effectiveness of pharmacological treatments for adolescents and adults with autism spectrum disorder. Autism 11(4):335-348

Cai B, Xu W, Bortnichak E, Watson D (2012) An algorithm to identify medical practices common to both the General Practice Research Database and The Health Improvement Network database. Pharmacoepidemiol Drug Saf 21:770-774

Cegedim Strategic Data Medical Research UK (2009) THIN Data from EPIC: a guide for researchers. November 2009

Chisholm J (1990) The Read clinical classification. BMJ 300:1092

Esbensen AJ, Greenberg JS, Seltzer MM, Aman MG (2009) A longitudinal investigation of psychotropic and non-psychotropic medication use among adolescents and adults with autism spectrum disorders. J Autism Dev Disord 39(9):1339-1349

Fleischhaker C, Heiser P, Hennighausen K, Herpertz-Dahlmann B, Holtkamp K, Mehler-Wex C, Rauh R, Remschmidt H, Schulz E, Warnke A (2006) Clinical drug monitoring in child and adolescent psychiatry: side effects of atypical neuroleptics. J Child Adolesc Psychopharmacol 16(3):308-316

Fombonne E, Heavey L, Smeeth L, Rodrigues LC, Cook C, Smith PG, Meng L, Hall AJ (2004) Validation of the diagnosis of autism in general practitioner records. BMC Publ Health 4:5

Frazier TW, Shattuck PT, Narendorf SC, Cooper BP, Wagner M, Spitznagel EL (2011) Prevalence and correlates of psychotropic medication use in adolescents with an autism spectrum disorder with and without caregiver-reported attention-deficit/hyperactivity disorder. J Child Adolesc Psychopharmacol 21(6):571-579

Green H, McGinnity A, Meltzer H, Ford T, Goodman R (2005) Mental health of children and young people in Great Britain, 2004. London: National Statistics Office, Controller of Her Majesty's Stationery Office

Handen BL, Johnson CR, Lubetsky M (2000) Efficacy of methylphenidate among children with autism and symptoms of attention-deficit hyperactivity disorder. J Autism Dev Disord 30(3):245-255

Knapp M, Romeo R, Beecham J (2009) Economic cost of autism in the UK. Autism 13(3):317-336

Leyfer OT, Folstein SE, Bacalman S, Davis NO, Dinh E, Morgan J, Tager-Flusberg H, Lainhart JE (2006) Comorbid psychiatric disorders in children with autism: interview development and rates of disorders. J Autism Dev Disord 36(7):849-861

Logan SL, Nicholas JS, Carpenter LA, King LB, Garrett-Mayer E, Charles JM (2012) High prescription drug use and associated costs among Medicaid-eligible children with autism spectrum disorders identified by a population-based surveillance network. Ann Epidemiol 22(1):1-8

Maguire A, Blak BT, Thompson M (2009) The importance of defining periods of complete mortality reporting for research using automated data from primary care. Pharmacoepidemiol Drug Saf 18(1):76-83

Mandell DS, Morales KH, Marcus SC, Stahmer AC, Doshi J, Polsky DE (2008) Psychotropic medication use among Medicaidenrolled children with autism spectrum disorders. Pediatrics 121(3):e441-e448. doi:10.1542/peds.2007-0984

Marcus RN, Owen R, Kame L, Manos G, McQuade RD, Carson WH, Aman MG (2009) A placebo-controlled, fixed-dose study of aripiprazole in children and adolescents with irritability associated with autistic disorder. J Am Acad Child Adolesc Psychiatry 48(11):1110-1119

McCarthy S, Wilton L, Murray ML, Hodgkins P, Asherson P, Wong IC (2012a) The epidemiology of pharmacologically treated attention deficit hyperactivity disorder (ADHD) in children, adolescents and adults in UK primary care. BMC Pediatr 12:78. doi:10.1186/14712431-12-78

McCarthy S, Wilton L, Murray ML, Hodgkins P, Asherson P, Wong IC (2012b) Persistence of pharmacological treatment into adulthood, in UK primary care, for ADHD patients who started treatment in childhood or adolescence. BMC Psychiatry 12(1):219, Epub ahead of print 
Morgan S, Taylor E (2007) Antipsychotic drugs in children with autism. BMJ 334:1069-1070

Oswald DP, Sonenklar NA (2007) Medication use among children with autism spectrum disorders. J Child Adolesc Psychopharmacol 17(3):348-355

Owen R, Sikich L, Marcus RN, Corey-Lisle P, Manos G, McQuade RD, Carson WH, Findling RL (2009) Aripiprazole in the treatment of irritability in children and adolescents with autistic disorder. Pediatrics 124(6):1533-1540

Rani F, Murray ML, Byrne PJ, Wong IC (2008) Epidemiologic features of antipsychotic prescribing to children and adolescents in primary care in the United Kingdom. Pediatrics 121(5):1002-1009

Rani FA, Byrne PJ, Murray ML, Carter P, Wong IC (2009) Paediatric Atypical Antipsychotic Monitoring Safety (PAMS) Study: pilot study in children and adolescents in secondary- and tertiary-care settings. Drug Saf 32(4):325-333

Rani FA, Byrne P, Cranswick N, Murray ML, Wong IC (2011) Mortality in children and adolescents prescribed antipsychotic medication: a retrospective cohort study using the UK general practice research database. Drug Saf 34(9):773-781

Research Units on Pediatric Psychopharmacology Autism Network (2002) Risperidone in children with autism and serious behavioral problems. N Engl J Med 347(5):314-321

Research Units on Pediatric Psychopharmacology Autism Network (2005) Randomized, controlled, crossover trial of methylphenidate in pervasive developmental disorders with hyperactivity. Arch Gen Psychiatry 62:1266-1274

Robb AS, Andersson C, Bellocchio EE, Manos G, Rojas-Fernandez C, Mathew S, Marcus R, Owen R, Mankoski R (2011) Safety and tolerability of aripiprazole in the treatment of irritability associated with autistic disorder in pediatric subjects (6-17 years old): results from a pooled analysis of 2 studies. Prim Care Companion CNS Disord 13(1). doi: 10.4088/PCC.10m01008gry

Sanchez LE, Campbell M, Small AM, Cueva JE, Armenteros JL, Adams PB (1996) A pilot study of clomipramine in young autistic children. J Am Acad Child Adolesc Psychiatry 35(4):537-544

Shattuck PT, Durkin M, Maenner M, Newschaffer C, Mandell DS, Wiggins L, Lee LC, Rice C, Giarelli E, Kirby R, Baio J, PintoMartin J, Cuniff C (2009) Timing of identification among children with an autism spectrum disorder: findings from a population- based surveillance study. J Am Acad Child Adolesc Psychiatry 48(5):474-483

Shea S, Turgay A, Carroll A, Schulz M, Orlik H, Smith I, Dunbar F (2004) Risperidone in the treatment of disruptive behavioral symptoms in children with autistic and other pervasive developmental disorders. Pediatrics 114(5):e634-e641, Epub 2004 Oct 18

Simonoff E (2012) Autism spectrum disorder: prevalence and cause may be bound together. Br J Psych 201:88-89

Simonoff E, Taylor E, Baird G, Bernard S, Chadwick O, Liang H, Whitwell S, Riemer K, Sharma K, Sharma SP, Wood N, Kelly J, Golaszewski A, Kennedy J, Rodney L, West N, Walwyn R, Jichi F (2012) Randomized controlled double-blind trial of optimal dose methylphenidate in children and adolescents with severe attention deficit hyperactivity disorder and intellectual disability. J Child Psychol Psychiatry. doi: 10.1111/j.1469-7610.2012.02569.x

Simonoff E, Pickles A, Charman T, Chandler S, Loucas T, Baird G (2008) Psychiatric disorders in children with autism spectrum disorders: prevalence, comorbidity, and associated factors in a population-derived sample. J Am Acad Child Adolesc Psychiatry 47(8):921-929

Souders MC, Mason TB, Valladares O, Bucan M, Levy SE, Mandell DS, Weaver TE, Pinto-Martin J (2009) Sleep behaviors and sleep quality in children with autism spectrum disorders. Sleep 32(12):1566-1578

Star K, Iessa N, Almandil NB, Wilton L, Curran S, Edwards IR, Wong IC (2012) Rhabdomyolysis reported for children and adolescents treated with antipsychotic medicines: a case series analysis. J Child Adolesc Psychopharmacol 22(6):440-451

White SW, Oswald D, Ollendick T, Scahill L (2009) Anxiety in children and adolescents with autism spectrum disorders. Clin Psychol Rev 29(3):216-229

Wijlaars LP, Nazareth I, Petersen I (2012) Trends in depression and antidepressant prescribing in children and adolescents: a cohort study in The Health Improvement Network (THIN). PLoS One 7 (3):e33181. doi:10.1371/journal.pone.0033181, Epub 2012 Mar 13

Williams PG, Woods C, Stevenson M, Davis DW, Radmacher P, Smith M (2012) Psychotropic medication use in children with autism in the Kentucky Medicaid population. Clin Pediatr (Phila) 51(10):923-927 